\title{
Evolution of Sequence Type 4821 Clonal Complex Meningococcal Strains in China from Prequinolone to Quinolone Era, 1972-2013
}

\author{
Qinglan Guo, ${ }^{1}$ Mustapha M. Mustapha, ${ }^{1}$ Mingliang Chen, Di Qu, Xi Zhang, \\ Min Chen, Yohei Doi, Minggui Wang, Lee H. Harrison
}

\begin{abstract}
The expansion of hypervirulent sequence type 4821 clonal complex (CC4821) lineage Neisseria meningitidis bacteria has led to a shift in meningococcal disease epidemiology in China, from serogroup A (MenA) to MenC. Knowledge regarding the evolution and genetic origin of the emergent MenC strains is limited. In this study, we subjected 76 CC4821 isolates collected across China during 1972-1977 and 2005-2013 to phylogenetic analysis, traditional genotyping, or both. We show that successive recombination events within genes encoding surface antigens and acquisition of quinolone resistance mutations possibly played a role in the emergence of CC4821 as an epidemic clone in China. MenC and MenB CC4821 strains have spread across China and have been detected in several countries in different continents. Capsular switches involving serogroups B and $C$ occurred among epidemic strains, raising concerns regarding possible increases in MenB disease, given that vaccines in use in China do not protect against MenB.
\end{abstract}

he incidence of meningococcal disease and Neisseria
meningitidis strain distribution vary over time, within and between countries and regions (1). Six serogroups (A, $\mathrm{B}, \mathrm{C} \mathrm{W}, \mathrm{X}$, and $\mathrm{Y}$ ) account for nearly all cases of invasive meningococcal disease (IMD) globally (1). Serogroup C (MenC) cases were rare in China until 2003-2005, when several MenC outbreaks were reported in Anhui Province (2-4). These outbreaks were caused by a previously unreported hypervirulent clonal complex (CC) 4821 lineage (5). A pharyngeal carriage survey and national public health surveillance during 2004-2005 identified CC4821 among sporadic IMD case-patients and asymptomatic

Author affiliations: Fudan University Huashan Hospital, Shanghai, China (Q. Guo, M. Wang); University of Pittsburgh School of Medicine, Pittsburgh, Pennsylvania, USA (M.M. Mustapha, Y. Doi, L.H. Harrison); Shanghai Municipal Center for Disease Control and Prevention, Shanghai (M. Chen, X. Zhang, M. Chen); Fudan University Shanghai Medical College, Shanghai (D. Qu)

DOI: https://doi.org/10.3201/eid2404.171744 carriers across 11 provinces, demonstrating the wide geographic distribution of CC4821 (2). During 2005-2012, MenC CC4821 became the leading cause of endemic meningococcal disease in China (6). Further analyses of historic isolate collections identified MenB and MenC CC4821 from carriage surveys in the 1970s $(2,7)$. These studies demonstrated that CC4821 had been mostly associated with asymptomatic carriage over several decades before it emerged as a main cause of $\operatorname{IMD}(2,7,8)$. Also, our recent analyses of quinolone resistance among historic meningococcal isolate collections in China found a substantial temporal shift toward increased quinolone nonsusceptibility from the prequinolone era (before $\approx 1985$ ) to the quinolone era (none versus $>70 \%$ ), particularly within hypervirulent CC4821 and CC5 lineages (7). Such findings support the hypothesis that quinolone resistance could have played a role in the emergence of MenC CC4821 outbreaks in China.

Meningococci have a dynamic genome that evolves rapidly through point mutations and frequent recombination. Such genetic changes give rise to strains with novel capsular or other major surface antigens that evade existing population immunity (9). A study by Zhu et al. found extensive genomic variation among 22 CC4821 invasive and carriage isolates from 12 provinces in China during 2005-2011 (8). In that study, CC4821 isolates belonged to 2 distinct phylogenetic groups, and results indicated that group 1, containing the epidemic reference strain 053442 , might be more invasive than group 2 and that MenB and MenC coexisted within both groups 1 and $2(8)$.

Our study describes phylogenetic relationships within a collection of historic and current isolates from Shanghai in the prequinolone and quinolone eras and explores how the isolates fit into the larger genetic profile of CC4821 from China (8). We aimed to shed light on the genomic factors underlying the abrupt transition of this lineage from a minority strain to a leading cause of endemic disease and outbreaks.

${ }^{1}$ These authors contributed equally to this article. 


\section{Materials and Methods}

\section{Strain Selection and Molecular Typing}

A total of 374 meningococcal isolates were collected from IMD case-patients, close contacts, and asymptomatic carriers during pharyngeal carriage surveys and routine, laboratory-based public health surveillance conducted during 1965-1985 and 2005-2013 (7). The interruption during 1986-2004 was because of the decreased incidence of meningococcal cases and the increased ability to identify $N$. meningitidis in hospitals, necessitating fewer isolates to be referred (7). In all, 52 CC4821 isolates were identified and underwent molecular characterization using traditional PCR sequencing of the porA, porB, fetA, fHbp, nadA, $n h b a$, and gyrA genes and pulsed-field gel electrophoresis as described previously $(7,10,11)$. We selected 8 of these 52 isolates, representing strains from different periods, serogroups, or pulsed-field gel electrophoresis groups, for whole-genome sequencing (WGS) and in-depth phylogenetic analysis. We downloaded assembled contiguous genome sequences (contigs) for 24 additional genome sequences from previous studies of CC4821 in China from GenBank (NM11003, accession no. NZ_ANBU00000000) $(5,8)$. Therefore, a total of 32 whole-genome sequences underwent core genome phylogenetic analyses for this study (online Technical Appendix Table 1, https://wwwnc.cdc. gov/EID/article/24/4/17-1744-Techapp1.pdf).

\section{Genome Sequencing and Assembly}

We performed single molecule real-time sequencing (PacBio; Pacific Biosciences, Menlo Park, CA, USA) on 4 CC4821 isolates (NM040, NM062, NM205, and NM323) and sequenced the remaining 4 isolates (NM001, NM050, NM193, and NM313) by using Illumina HiSeq paired-end sequencing (Illumina, San Diego, CA, USA). We assembled PacBio genomes by using HGAP 4.0 (https://github. com/PacificBiosciences/Bioinformatics-Training/wiki/ HGAP) and Illumina genomes by using SPAdes 3.7 (12). We annotated assembled contigs by using the Prokka v1.11 (13) pipeline and submitted them to the PubMLST Neisseria genome database (http://pubmlst.org/neisseria) with ID numbers 41414-41421, where allelic numbers were assigned to all identified genes (14).

\section{Phylogenetic Analyses}

We aligned assembled genomes $(\mathrm{n}=32)$ and produced a core genome phylogenetic tree with 1,000 rapid bootstrap replicates by using RAxML and Mauve 2.3 as previously described (15-17). Serogroup A reference genome Z2491 was included as an outgroup (18). We aligned gene sequences selected for focused analyses with MEGA 5.2 (http://www. megasoftware.net) and constructed maximum-likelihood phylogenetic trees under the HKY model of evolution (19).
We included comparison sequences from a global collection of 133 genomes from the PubMLST Neisseria genome database representing all major invasive disease lineages in some of these phylogenetic analyses as references.

\section{Recombination}

We assessed recombination by using ClonalFrameML and Gubbins $(20,21)$. We then mapped the recombination to the CC4821 reference genome 053442 (online Technical Appendix).

\section{Gene Content}

We assessed gene content by using the Roary 3.6 ortholog clustering program (22), which identifies presence or absence of orthologous gene sequences using a cutoff of $90 \%$ sequence identity $(-\mathrm{i}=90)$. We defined core genes as genes present in $\geq 90 \%$ of the genomes. When comparing the gene content of 2 groups of genomes, we defined a gene as specific to that group if it was present in $\geq 90 \%$ of the genomes in the group and in $<20 \%$ of the genomes in the comparison group. We downloaded gene functional annotation from the COG database (23) and compared genes containing recombinant sequences with nonrecombinant ones based on the major COG classes (cellular processes and signaling, information storage and processing, metabolism, poorly characterized) using uncorrected $\chi^{2}$ tests.

\section{Results}

Out of 52 Shanghai isolates, 18 (34\%) were from 1972 1977 (3 IMD isolates and 15 asymptomatic carriage isolates), and the remaining 34 (23 IMD isolates, 6 isolates from close contacts, and 5 carriage isolates) were isolated during 2005-2013. Most (56\%) Shanghai isolates were serogroup C, $42 \%$ were serogroup B, and 1 was nongroupable (online Technical Appendix Table 2).

\section{Phylogenetic Analyses of CC4821 Isolates in China}

We conducted comparative genome analyses for 8 isolates from Shanghai and 24 publicly available genomes from across China (Figure 1). Among the 8 newly sequenced isolates, 4 were from asymptomatic carriers from 1972-1977 (NM193, NM205, NM313, and NM323), 3 were IMD isolates from 2005-2011 (NM001, NM062, and NM040), and 1 was from an asymptomatic contact of a meningitis patient (NM050). The remaining 24 genome sequences included invasive and carriage CC4821 isolates from 12 provinces across China from 2004-2011, as characterized in previous studies $(4,8)$. Overall, MenC represented 66\% (21/32) of isolates that we analyzed, and the remaining $34 \%(11 / 32)$ belonged to MenB.

The core genome phylogenetic tree classified CC4821 into 2 distinct groups (Figure 1). Group 1 consisted of several isolates that were very closely related; this group con- 


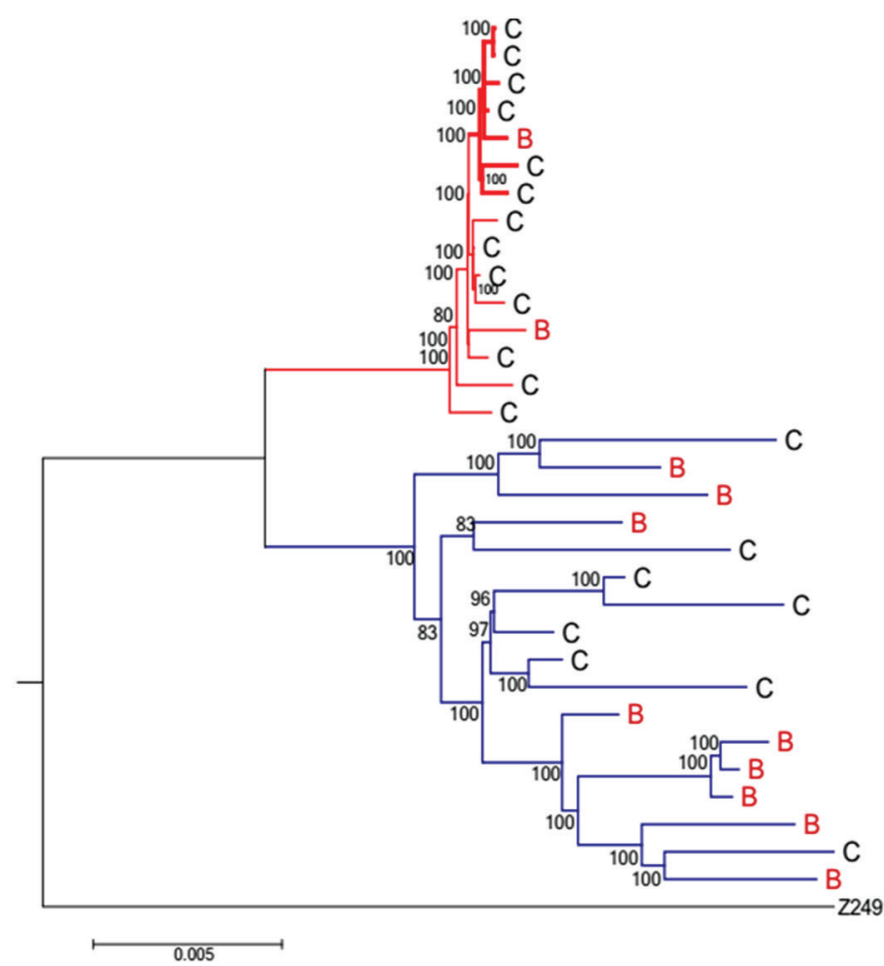

\begin{tabular}{|c|c|c|c|c|c|c|c|c|c|}
\hline Isolate & Year & $\mathrm{Sg}$ & ST & CC & $\begin{array}{l}\text { Province/ } \\
\text { city }\end{array}$ & $\begin{array}{l}\text { PorA } \\
\text { VR1 }\end{array}$ & $\begin{array}{l}\text { PorA } \\
\text { VR2 - }\end{array}$ & $\begin{array}{l}\text { gyrA } \\
\text { allele }\end{array}$ & Disease \\
\hline NM330505 & 2005 & c & 4821 & 4821 & Zhejiang & $7-2$ & 14 & 71(T911) & + \\
\hline NMO01 & 2005 & c & 4821 & 4821 & Shanghai & $7-2$ & 14 & 71(T911) & + \\
\hline 053442 & 2004 & c & 4821 & 4821 & Anhui & $7-2$ & 14 & 71(T911) & + \\
\hline NM220601 & 2006 & c & 4821 & 4821 & Jilin & $7-2$ & 14 & 71(T911) & + \\
\hline NM341215 & 2011 & B & 4821 & 4821 & Anhui & $7-2$ & 14 & 71(T911) & + \\
\hline NM320501 & 2005 & c & 4820 & 4821 & Jiangsu & $7-2$ & 14 & 71(T911) & + \\
\hline NM062 & 2011 & C & 4821 & 4821 & Shanghai & $7-2$ & 14 & 71 (T911) & + \\
\hline NM420718 & 2007 & c & 11920 & 4821 & Hubei & $12-1$ & $16-8$ & 71(T911) & + \\
\hline NM11003 & 2011 & c & 4821 & 4821 & Guangdong & $12-1$ & $16-8$ & 71(T911) & + \\
\hline NM100603 & 2005 & c & 4821 & 4821 & Beijing & $12-1$ & $16-8$ & 71(T911) & + \\
\hline NM100601 & 2005 & c & 10737 & 4821 & Beijing & $12-1$ & $16-8$ & 71(T911) & + \\
\hline NM440902 & 2008 & B & 4821 & 4821 & Guangdong & 20 & 23-1 & 71(T911) & + \\
\hline NM320503 & 2005 & c & 4821 & 4821 & Jiangsu & 20 & 23-9 & 71(T91I) & + \\
\hline NM130803 & 2008 & c & 6928 & 4821 & Hebei & 20 & $23-1$ & 71(T91I) & \\
\hline NM131148 & 2011 & $c$ & 9936 & 4821 & Hebei & 20 & 23-28 & 71(T911) & \\
\hline NM100572 & 2005 & c & 5610 & 4821 & Beijing & 20 & 23 & 12 & \\
\hline NM340552 & 2005 & B & 4897 & 4821 & Anhui & 21-2 & 28 & 12 & \\
\hline NM34173 & 2011 & B & 9477 & 4821 & Guangxi & 20 & 23-18 & 171(T911) & \\
\hline NM421007 & 2009 & B & 4821 & 4821 & Hubei & $12-11$ & $16-8$ & 12 & \\
\hline NM100514 & 2005 & c & 4832 & 4821 & Beijing & 20 & $23-1$ & 203(T911) & \\
\hline NM193 & 1972 & c & 3436 & 4821 & Shanghai & 20-3 & $23-1$ & 12 & \\
\hline NM440501 & 2005 & c & 4831 & 4821 & Guangdong & $20-3$ & 23 & 12 & + \\
\hline NM205 & 1973 & c & 4821 & 4821 & Shanghai & 20 & $23-2$ & 4 & \\
\hline NM313 & 1977 & c & 4821 & 4821 & Shanghai & 20 & 23-1 & 12 & \\
\hline NM360624 & 2006 & c & 5473 & 4821 & Jiangxi & UA & UA & 114(T911) & \\
\hline NM323 & 1977 & B & 5798 & 4821 & Shanghai & 20 & 23 & 12 & \\
\hline NM040 & 2007 & B & 5798 & 4821 & Shanghai & 20 & $13-1$ & 23 & + \\
\hline NM311112 & 2011 & B & 5798 & 4821 & Shanghai & 20 & $13-1$ & 12 & + \\
\hline NM050 & 2009 & B & 5798 & 4821 & Shanghai & 20 & $13-2$ & 71(T911) & \\
\hline NM100530 & 2005 & B & 5614 & 4821 & Beijing & $20-3$ & 23 & 173(T91V) & \\
\hline NM370601 & 2005 & c & 3200 & 4821 & Shandong & 20 & 23 & UA(T911) & + \\
\hline NM321114 & 2011 & B & 3200 & 4821 & Jiangsu & 20 & 23-3 & 175(T911) & + \\
\hline
\end{tabular}

Epidemic clone

Group 1

Group 2

Figure 1. Core genome phylogenetic tree showing relationships between Neisseria meningitidis serogroups C and B CC4821 strains, China, 1972-2011. The strains cluster within 1 of 2 distinct phylogenetic groups, group 1 and group 2 . Within group 1 is an antigenically distinct clonal group (epidemic clone) containing outbreak-associated strains. Tree is rooted using serogroup A reference strain (Z2491) as an outgroup. Maximum-likelihood phylogenetic trees of aligned core genome sequences were generated under a general time reversible model of evolution with gamma rate heterogeneity, with 1,000 rapid bootstrap replicates represented as a percentage. Only node labels with $>80 \%$ bootstrap support are shown. Strain type and date and place of isolation are shown; + indicates strains isolated from invasive disease cases. Resistant point mutations on the T91 position of gyrA gene are shown alongside data on gyrA allele designation. Scale bar represents total substitutions per site. CC, clonal complex; PorA VR, outer membrane protein PorA variable regions; Sg, serogroup; ST, sequence type.

tained 15 isolates from 2004 to 2011, and most were from IMD case-patients. In addition, the epidemic reference strain 053442 clustered with a small group of highly similar group 1 isolates. This subgroup is defined as the epidemic clone based on core genome phylogenetic analyses and antigen gene profile (described hereafter). A second more diverse phylogenetic group (group 2) consisted of isolates from Shanghai during 1972-1977 and more recent ones collected from 9 provinces during 2005-2011. In contrast to group 1, only $29 \%$ of group 2 isolates were from IMD case-patients (Figure 1). MenB was found in both groups and was interspersed with MenC genomes. Two of $11 \mathrm{MenB}$ isolates belonged to group 1, the remainder to group 2 (Figure 1; online Technical Appendix Table 1).

\section{Characterization of Antigen Gene Content}

We examined 32 isolates that had undergone WGS to identify the major antigen gene (PorA VR1 and VR2, porB, FetA, $n h b a$, and $f H b p$ ) alleles that corresponded to the epidemic strain, group 1 or group 2, as determined by the core genome phylogenetic analysis (Figures 1,2; online Technical Appendix Table 1, Figures 1-5). nadA was missing in all CC4821 study genomes. Group 1 genomes had diverse $f H b p$ and porA alleles. All 15 group 1 isolates contained the porB 3-48 and FetA F3-3 alleles, and nhba allele 124. The epidemic clone contained a few highly related porA alleles that all encoded unique, conserved porA variable regions (PorA VR1 and VR2: P1.7-2, 14). Group 2 isolates had the most antigen gene diversity, containing 5-12 different alleles for each antigen-encoding gene at the nucleotide level and no clear predominance of any single allelic profile. We observed little overlap between the antigen gene allelic profiles in groups 1 and 2. Only 2 of 13 group 1 alleles were also found in group 2. None of the antigen gene alleles found in the epidemic clone was present in group 2, suggesting that the epidemic clone had a nonoverlapping repertoire of antigens compared with historic and current group 2 isolates (Figure 2; online Technical Appendix Table 1, Figures 1-5). Group 2 was predominantly associated with PorA P1.20 variants (82\%); PorB 3-229 (29\%); 


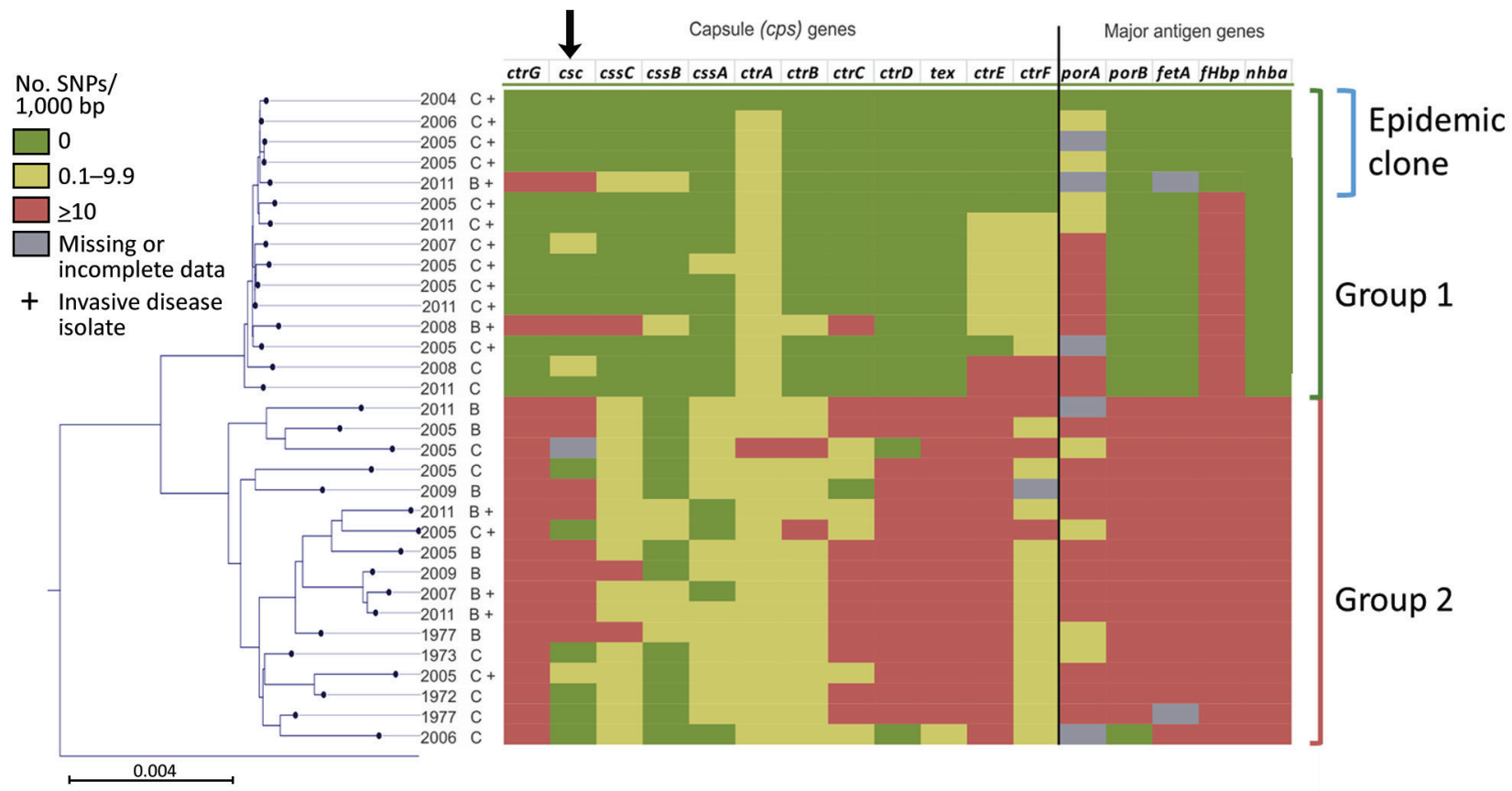

Figure 2. Genetic diversity within capsule and major antigen encoding genes among 32 Neisseria meningitidis clonal complex 4821 isolates, China, 1972-2011. Gene sequences from 32 clonal complex 4821 isolates were compared with the epidemic reference strain 053442 (the topmost isolate on the juxtaposed core genome phylogenetic tree). Scale bar represents total substitutions per site. SNP, single-nucleotide polymorphism.

FetA F3-9 (18\%) and FetA F5-2 (18\%); FHbp peptide 16 (65\%); and nhba 553 (41\%) (online Technical Appendix Table 1).

\section{Recombination Events Separating Group 1 and Group 2 Isolates}

We identified extensive recombination within CC4821 lineage, as detected by ClonalFrameML (20). The estimated rate of recombination relative to mutation $(\mathrm{R} / \Theta)$ for the entire CC4821 core genome was 1.37 (95\% CI 1.40-1.34), and the relative impact of recombination to mutation $(\mathrm{r} / \mathrm{m})$ was 20.83 , indicating that $\approx 21 \mathrm{nt}$ were acquired by recombination for every point mutation within the core genome of CC4821. Sublineage specific recombination rates $(\mathrm{R} / \Theta)$ were 1.79 (95\% CI 1.53-2.05) for group 1, 1.47 (95\% CI $1.15-1.79)$ for group 2 , and $0.5(95 \%$ CI $0.26-0.75)$ for the epidemic clone.

A total of 46 recombination fragments containing 120 genes were mapped to the node that separated group 1 from group 2, indicating that these recombination events could be linked to the divergence of group 1 and 2 sublineages (online Technical Appendix Table 3). Sequence alignment and phylogenetic analysis confirmed that the 46 genomic loci represented regions of marked sequence divergence, presumably caused by homologous recombination within a common ancestral genome. These 120 recombinant genes belonged to diverse functional categories; metabolism was the overrepresented functional assignment (51/120 [42.5\%]), compared with $28 \%$ of nonrecombinant genes $\left(\mathrm{p}<0.001\right.$ by $\chi^{2}$ test). Proportions of other functional groups were similar between recombinant and nonrecombinant genes.

\section{Recombination Events Unique to the Epidemic Clone}

The epidemic clone diverged from group 1 through several additional recombination events, affecting 24 genes across 7 genomic loci (Table). These genomic loci included capsule translocation genes ( $c t r E$ and $c t r F)$; major outer membrane protein (OMP) antigen genes por A, por B, and $\mathrm{fHbp}$; $p t s$ genes involved in carbohydrate transport and metabolism; and the $t k l$ locus encoding DNA polymerase and several metabolic enzymes.

\section{Gene Content}

We identified a total of 3,292 unique genes, of which 1,730 core genes were shared by most CC4821 genomes. Eleven genes were present in $\geq 90 \%$ of group 1 isolates but missing in most group 2 isolates. Genes that were predominantly found in group 1 included $l b p B$ encoding lactoferrin binding protein $\mathrm{B}, n h a P$ encoding $\mathrm{Na}^{+} / \mathrm{K}^{+}$antiporter, and genes encoding several putative enzymes and 4 hypothetical proteins whose functions are unknown (online Technical Appendix Table 4). These genes were spread across several locations on the reference genome 053442 , suggesting that they were acquired separately rather than in a single event. The epidemic clone had no noticeable gene gain or loss compared with other group 1 isolates. 
Table. Recombinant genes unique to the Neisseria meningitidis clonal complex 4821 epidemic clone, China, 1972-1977 and 2005-2013*

\begin{tabular}{|c|c|c|}
\hline Gene ID & Gene name & Annotation \\
\hline NMCC_0090 & ctrE & Polysialic acid capsule modification protein LipA \\
\hline NMCC_0091 & ctrF & Polysialic acid capsule modification protein LipB \\
\hline NMCC 0136 & & Putative RmuC-like protein \\
\hline NMCC_0137 & & Putative metallo-dependent hydrolase \\
\hline NMCC_0138 & & Putative periplasmic DNA ligase (polydeoxyribonucleotide synthase [ATP]) \\
\hline NMCC_0140 & ptsIIA & Phosphotransferase system, enzyme IIA (protein IIA) \\
\hline NMCC_0141 & ptsH & Phosphocarrier protein HPr (phosphotransferase system, histidine-containing protein) \\
\hline NMCC_0142 & ptsl & $\begin{array}{l}\text { Phosphoenolpyruvate-protein phosphotransferase (phosphotransferase system, } \\
\text { enzyme I; protein I) }\end{array}$ \\
\hline NMCC_0158 & porB & Major outer-membrane protein P.IB (protein IB; PIB; porin) \\
\hline NMCC_0350 & & Putative peptidase \\
\hline NMCC_0351 & $f H b p$ & Factor H binding lipoprotein (lipoprotein GNA1870) \\
\hline NMCC_0352 & fba & Fructose-bisphosphate aldolase \\
\hline NMCC_1338 & porA & Major outer-membrane protein P.IA (protein IA; PIA; porin) \\
\hline NMCC_1341 & greA & Transcription elongation factor GreA (transcript cleavage factor GreA) \\
\hline NMCC_1342 & $\operatorname{aro} A$ & $\begin{array}{l}\text { 3-phosphoshikimate 1-carboxyvinyltransferase (5-enolpyruvylshikimate-3-phosphate } \\
\text { synthase; EPSP synthase; EPSPS) }\end{array}$ \\
\hline NMCC_1343 & & Conserved hypothetical lipoprotein \\
\hline NMCC_1363 & & Putative DnaQ-like exonuclease \\
\hline NMCC_1364 & & $\operatorname{Arg} \operatorname{tRNA}$ \\
\hline NMCC_1365 & & Glu tRNA \\
\hline NMCC_1366 & & Putative dioxygenase \\
\hline NMCC_1367 & & Conserved hypothetical membrane protein \\
\hline NMCC_1368 & & Putative ferredoxin \\
\hline NMCC_1370 & tkt & Transketolase \\
\hline NMCC_2038 & fmt & Methionyl-tRNA formyltransferase \\
\hline
\end{tabular}

\section{Capsular Gene cps Cluster}

The cps cluster of $N$. meningitidis consists of 6 regions (D-A-C-E-D'-B) required for capsule biosynthesis (region A), transport (region C), and translocation (region B) (24). The group 1 isolates were characterized by a novel capsule region A (ctrG4-cssE1-csc1-cssC3-cssB1-cssA3), associated with region $\mathrm{C}(\mathrm{ctr} A 5-\mathrm{ctrB} 1-\operatorname{ctr} C 4-\mathrm{ctrD})$ and region E (tex-orf1-orf2) (online Technical Appendix Table 1). Nine of 15 regions A-C-E identified among group 1 isolates were identical, and another 4 were almost identical (1-bp variation in $\csc , \operatorname{css} A$, or $\operatorname{ctr} A$ over 14,489 bp). Only 2 diverged because of the allelic replacement containing the serogroup-specific polysialyltransferase gene $(c s b)$, resulting in capsular switches involving serogroups B and C (NM341215 and NM440902) (online Technical Appendix Table 2). Group 2 had substantial genetic diversity within the cps cluster, with no 2 isolates possessing an identical cps gene allelic profile (Figure 2).

\section{Characterization of CC4821 Isolates from Shanghai}

Our results classified 52 CC4821 isolates from Shanghai into epidemic clone or groups 1 and 2 on the basis of associated porA, porB, fetA, fHbp, and nhba antigen genes defined by WGS (Figure 1; online Technical Appendix Table 2). A substantial proportion of strains in Shanghai (23/52 [44\%]) belonged to group 1, with $0-1$ antigen gene differences (Figure 1; online Technical Appendix Table 2, Figures 1-5). Shanghai group 1 isolates were exclusively isolated during 2005-2013; were MenC (19/23), MenB $(3 / 23)$, or nongroupable (1/23); and were isolated from IMD case-patients (18/23), close contacts of IMD casepatients $(4 / 23)$, or an asymptomatic carrier $(1 / 23)$. Within group 1 , a total of 16 isolates $(16 / 23$ [70\%]) contained PorA P1.7-2,14 and were consistent with the epidemic clone by all 5 antigen genes. Twenty-nine (29/52 [56\%]) Shanghai isolates belonged to group 2 and differed from group 1 by $4-5$ out of 5 antigen loci. Shanghai group 2 was dominated by carriage isolates $(21 / 29$ [72\%]) from 1972-1977 and 2005-2013. MenB equaled or outnumbered MenC among historic (9/18 [50\%]) and recent (10/11 [91\%]) group 2 CC4821 isolates. MenB strains in group 1 possessed $\mathrm{fHbp} 498$ or 22 encoding peptides 1.80 or 2.22 (subvariants belonging to FHbp variant group 1 or 2), as most (17/19 [89\%]) of MenC epidemic clones did (online Technical Appendix Tables 1, 2).

All group 1 isolates contained fluoroquinolone-resistant gyrA allele 71 (corresponding to allele $\mathrm{R} 1$ in our previous study [7]), which was generated by a nonsynonymous mutation of fluoroquinolone-susceptible gyrA allele 12 (allele S1), creating an amino acid substitution of T91I. The gyrA allele $12 / \mathrm{S} 1$ was carried by $76 \%(22 / 29)$ of the group 2 strains (online Technical Appendix Table 2). In contrast, only 4 of 29 group 2 isolates from Shanghai and another 6 publicly available group 2 genomes contained resistant gyrA alleles, which were different from each other. Group 2 isolates with genotypic resistance to quinolones were 
genetically highly diverse as evidenced by diverse sequence types, different antigen gene profiles, and expression of both MenB and MenC capsules. However, all quinoloneresistant isolates were from 2005-2013 (Figure 1; online Technical Appendix Tables 1,2).

\section{Discussion}

This study presents detailed genomic analyses of the current major endemic meningococcal disease lineages in China, serogroups C and B CC4821. Phylogenetic analyses have suggested that the epidemic clone (corresponding to the epidemic China ${ }^{\mathrm{CC} 4821-\mathrm{R} 1-\mathrm{C} / \mathrm{B}}$ clone described in our previous work [7)]) responsible for most recent CC4821 disease cases was nested within a distinct phylogenetic group (group 1), consistent with recent clonal expansion of a genetically distinct strain. In contrast, group 2 was temporally and genetically more diverse and accounted for a smaller proportion of IMD cases, as evidenced by the preponderance of asymptomatic carriage isolates within this group (8). Group 1 isolates were first identified during MenC outbreaks during 2003-2005, whereas group 2 isolates were mostly associated with carriage from the 1970s to 2013 (7).

This work adds to the description of CC4821 by a previous study ( 8 ) by demonstrating that, within group 1, a genetically distinct clone exists that shares the por $A$ antigenic formula P1.7-2,14 and comprises strains associated with outbreaks and a large proportion of epidemic disease cases. This finding is in keeping with surveillance studies reporting MenC strains during 2005-2012 indicating that 55\% of 238 confirmed meningococcal disease cases and $84 \%$ of 131 MenC strains belong to CC4821 with porA $\mathrm{P} 1.7-2,14$ (6), suggesting that the expansion of CC4821 was caused by clonal expansion of this antigenic type. Genomic analyses demonstrated that the epidemic CC4821 lineage had undergone 2 crucial genetic events compared with historic asymptomatic carriage isolates. First, CC4821 diverged into 2 major sublineages through extensive recombination events predominantly affecting genes involved in metabolic functions. Such extensive allelic exchanges might have enhanced the transmission fitness or the invasive potential within group 1. Second, a virulent, antigenically unique, epidemic strain emerged from within group 1 in a second set of more focused recombination events affecting major antigen genes, porA, por $B$, $f H b p$, capsule genes $c t r E$ and $c t r F$, and several metabolic genes associated with oxidative phosphorylation and glycolytic processes. These genetic changes possibly account for the rapid dissemination and increased invasive potential of the epidemic clone.

This work also adds to the evidence that the emergence and persistence of virulent meningococcal strains occurs through the introduction of a novel antigenic variant in an immunologically naive population $(9,15,25-27)$. In addition, even though a few major antigen gene repertoires mediate the microevolution of virulent meningococcal strains, a larger and more assorted number of metabolic genes might be involved in the divergence of sublineages. Additional research is needed to elucidate the intricate interactions between various metabolic pathways in the fitness and virulence potential of meningococci.

Selection pressure of fluoroquinolones might also affect the evolution and adaption of meningococcal strains, as evidenced by various resistant gyrA alleles recovered in many meningococcal lineages and singletons only in the quinolone era in China $(7,28)$. All of the group 1 CC4821 strains contain gyrA allele 71/R1, in contrast to various resistant gyrA alleles in group 2, suggesting acquisition of this trait by group 1 at an early stage in evolution. The gyr $A$ allele $71 / \mathrm{R} 1$ derived from gyrA allele $12 / \mathrm{S} 1$, which was possessed by most of the group 2 strains both in prequinolone and quinolone eras, indicating their common origin. The precise role of quinolone resistance in the emergence of the epidemic clone requires further study.

The relative rates and impact of recombination within the CC4821 lineage were considerably higher than previous estimates that examined recombination across 7 housekeeping genes (29-31). We identified 4,026 recombination events and 21 recombinant single-nucleotide polymorphisms (SNPs) for every point mutation within CC4821. Estimates of meningococcal recombination rates from whole-genome sequence data are limited. A study of MenA CC5 strains in Africa (27), an epidemic lineage notable for relatively low genetic diversity, found 34 recombinant sequences and 12 recombinant SNPs for every point mutation. A study of recombination rates within conserved multilocus sequence type loci among carriage isolates from the Czech Republic in 1993 found 6.2-16.8 SNPs caused by recombination for each point mutation (29). These data suggest that although homologous recombination is a shared mechanism for meningococcal strain emergence and persistence $(29,31-33)$, the frequency and extent of recombination likely differs substantially between lineages.

High rates of recombination have led to multiple distinct capsular switch strains expressing group B and C capsule, as described in this study, and serogroup W $(8,34)$. Both MenB and MenC were phylogenetically diverse and interspersed within both groups 1 and 2, suggesting multiple distinct capsular switches between these serogroups rather than clonal expansion of a single capsular switch strain $(35,36)$. Moreover, recombination events that led to no apparent change in capsular phenotype were also prevalent.

Wide geographic and temporal spread of MenB strains in China is of concern given that vaccines currently in use do not protect against MenB disease. Furthermore, marked heterogeneity exists among both MenB and MenC strains within the gene encoding FHbp, a key component of MenB vaccines. 
At the time of its emergence, CC4821 was confined to China and Taiwan (37). However, review of the PubMLST Neisseria genome database suggests a recent increase in the geographic spread of CC4821. CC4821 isolates were reported from a small number of asymptomatic carriers in Brazil (2014) $(38,39)$ and Australia (2012-2017), as well as isolated disease cases and carriers from France (20092011), the United Kingdom (2011-2014), the United States (2007-2016), and India (2017) (14). A recent case report of a quinolone-resistant sequence type 4821 strain from Japan in a patient with no history of foreign travel also suggests local transmission of CC4821 in Japan (40). Global CC4821 carriage and IMD isolates on the PubMLST Neisseria database were antigenically diverse, containing PorA types associated with both group 1 and 2 strains, and expressed both serogroup C and B capsules. This finding suggests low-level dissemination of CC4821 strains with diverse virulence and antigenic types as opposed to clonal spread of a single epidemic strain. This pattern is in contrast to the pandemic spread of highly clonal serogroup A epidemic strains from China from the 1960s through the 1990s (25). Genome-based surveillance of these global CC4821 strains is needed to monitor the global spread of this clonal lineage.

Our study is limited by lack of isolates before the 1970s and during 1986-2004; isolates from those periods might have provided a clearer picture of the multiple evolutionary steps that led to the epidemic CC4821 clone. Also, the high frequency of recombination within capsular genes makes it difficult to accurately determine the direction of capsular switch.

In summary, we have presented detailed genomic analysis of a major hypervirulent meningococcal lineage associated with MenC and MenB in China and identified key genomic factors that might have led to the emergence and persistence of MenC in China. The potential emergence of MenB is of public health concern. Strengthened laboratory surveillance for disease cases and carefully planned carriage surveys are needed to monitor global trends, detect outbreaks, and inform immunization policies.

\section{Acknowledgments}

We thank Jane Marsh for providing thoughtful comments on an early draft of this manuscript.

This study made use of the PubMLST Neisseria genome database (http://pubmlst.org/neisseria), developed by Keith Jolley and hosted by the University of Oxford. The development of this site has been funded by the Wellcome Trust and European Union. This study also made use of the Meningitis Research Foundation Meningococcus Genome Library (http://www. meningitis.org/research/genome), developed by Public Health England, the Wellcome Trust Sanger Institute, and the
University of Oxford as a collaboration. The project is funded by the Meningitis Research Foundation.

This work was supported by grants from the National Natural Science Foundation of China $(81673479,81120108024$, and 81601801); the Shanghai Pujiang Program (16PJD010); the Shanghai Rising-Star Program (17QA1403100); the Shanghai Medical Health Plan for Outstanding Young Talents (2017YQ039); and the 4th Three-Year Action Plan for Public Health of Shanghai (GWTD2015S01).

\section{About the Author}

Dr. Guo is an associate professor in the Institute of Antibiotics, Huashan Hospital, Fudan University. Her research interests include mechanisms of antimicrobial resistance in clinical isolates.

\section{References}

1. Halperin SA, Bettinger JA, Greenwood B, Harrison LH, Jelfs J, Ladhani SN, et al. The changing and dynamic epidemiology of meningococcal disease. Vaccine. 2012;30(Suppl 2):B26-36. http://dx.doi.org/10.1016/j.vaccine.2011.12.032

2. Zhang X, Shao Z, Yang E, Xu L, Xu X, Li M, et al. Molecular characterization of serogroup $\mathrm{C}$ Neisseria meningitidis isolated in China. J Med Microbiol. 2007;56:1224-9. http:/dx.doi.org/ 10.1099/jmm.0.47263-0

3. Ni JD, Jin YH, Dai B, Wang XP, Liu DQ, Chen X, et al. Recent epidemiological changes in meningococcal disease may be due to the displacement of serogroup A by serogroup C in Hefei City, China. Postgrad Med J. 2008;84:87-92. http://dx.doi.org/10.1136/ pgmj.2007.065680

4. Shao Z, Li W, Ren J, Liang X, Xu L, Diao B, et al. Identification of a new Neisseria meningitidis serogroup C clone from Anhui Province, China. Lancet. 2006;367:419-23. http://dx.doi.org/ 10.1016/S0140-6736(06)68141-5

5. Peng J, Yang L, Yang F, Yang J, Yan Y, Nie H, et al. Characterization of ST-4821 complex, a unique Neisseria meningitidis clone. Genomics. 2008;91:78-87. http://dx.doi.org/10.1016/j.ygeno.2007.10.004

6. Zhou H, Shan X, Sun X, Xu L, Gao Y, Li M, et al. Clonal characteristics of invasive Neisseria meningitidis following initiation of an A + C vaccination program in China, 2005-2012. J Infect. 2015;70:37-43. http://dx.doi.org/10.1016/j.jinf.2014.07.022

7. Chen M, Guo Q, Wang Y, Zou Y, Wang G, Zhang X, et al. Shifts in the antibiotic susceptibility, serogroups, and clonal complexes of Neisseria meningitidis in Shanghai, China: a time trend analysis of the pre-quinolone and quinolone eras. PLoS Med. 2015;12:e1001838. http://dx.doi.org/10.1371/journal.pmed.1001838

8. Zhu B, Xu Z, Du P, Xu L, Sun X, Gao Y, et al. Sequence type 4821 clonal complex serogroup B Neisseria meningitidis in China, 1978-2013. Emerg Infect Dis. 2015;21:925-32. http://dx.doi.org/ 10.3201/eid2106.140687

9. Harrison LH, Jolley KA, Shutt KA, Marsh JW, O’Leary M, Sanza LT, et al.; Maryland Emerging Infections Program. Antigenic shift and increased incidence of meningococcal disease. J Infect Dis. 2006;193:1266-74. http://dx.doi.org/10.1086/501371

10. Thompson EA, Feavers IM, Maiden MC. Antigenic diversity of meningococcal enterobactin receptor FetA, a vaccine component. Microbiology. 2003;149:1849-58. http://dx.doi.org/10.1099/ mic.0.26131-0

11. Urwin R, Fox AJ, Musilek M, Kriz P, Maiden MC. Heterogeneity of the PorB protein in serotype 22 Neisseria meningitidis. J Clin Microbiol. 1998;36:3680-2. 
12. Bankevich A, Nurk S, Antipov D, Gurevich AA, Dvorkin M, Kulikov AS, et al. SPAdes: a new genome assembly algorithm and its applications to single-cell sequencing. J Comput Biol. 2012;19:455-77. http://dx.doi.org/10.1089/cmb.2012.0021

13. Seemann T. Prokka: rapid prokaryotic genome annotation. Bioinformatics. 2014;30:2068-9. http://dx.doi.org/10.1093/ bioinformatics/btu153

14. Jolley KA, Maiden MC. BIGSdb: scalable analysis of bacterial genome variation at the population level. BMC Bioinformatics. 2010;11:595. http://dx.doi.org/10.1186/1471-2105-11-595

15. Mustapha MM, Marsh JW, Krauland MG, Fernandez JO, de Lemos APS, Dunning Hotopp JC, et al. Genomic epidemiology of hypervirulent serogroup W, ST-11 Neisseria meningitidis. EBioMedicine. 2015;2:1447-55. http://dx.doi.org/10.1016/ j.ebiom.2015.09.007

16. Stamatakis A. RAxML version 8: a tool for phylogenetic analysis and post-analysis of large phylogenies. Bioinformatics. 2014;30:1312-3. http://dx.doi.org/10.1093/bioinformatics/btu033

17. Darling AE, Mau B, Perna NT. progressiveMauve: multiple genome alignment with gene gain, loss and rearrangement. PLoS One. 2010;5:e11147. http://dx.doi.org/10.1371/journal.pone.0011147

18. Parkhill J, Achtman M, James KD, Bentley SD, Churcher C, Klee SR, et al. Complete DNA sequence of a serogroup A strain of Neisseria meningitidis Z2491. Nature. 2000;404:502-6. http://dx.doi.org/10.1038/35006655

19. Tamura K, Peterson D, Peterson N, Stecher G, Nei M, Kumar S. MEGA5: molecular evolutionary genetics analysis using maximum likelihood, evolutionary distance, and maximum parsimony methods. Mol Biol Evol. 2011;28:2731-9. http://dx.doi.org/ 10.1093/molbev/msr121

20. Didelot X, Wilson DJ. ClonalFrameML: efficient inference of recombination in whole bacterial genomes. PLOS Comput Biol. 2015;11:e1004041. http://dx.doi.org/10.1371/journal.pcbi.1004041

21. Croucher NJ, Page AJ, Connor TR, Delaney AJ, Keane JA, Bentley SD, et al. Rapid phylogenetic analysis of large samples of recombinant bacterial whole genome sequences using Gubbins. Nucleic Acids Res. 2015;43:e15. http://dx.doi.org/10.1093/nar/ gku1196

22. Page AJ, Cummins CA, Hunt M, Wong VK, Reuter S, Holden MT, et al. Roary: rapid large-scale prokaryote pan genome analysis. Bioinformatics. 2015;31:3691-3. http://dx.doi.org/10.1093/ bioinformatics/btv421

23. Tatusov RL, Galperin MY, Natale DA, Koonin EV. The COG database: a tool for genome-scale analysis of protein functions and evolution. Nucleic Acids Res. 2000;28:33-6. http://dx.doi.org/ 10.1093/nar/28.1.33

24. Harrison OB, Claus H, Jiang Y, Bennett JS, Bratcher HB, Jolley KA, et al. Description and nomenclature of Neisseria meningitidis capsule locus. Emerg Infect Dis. 2013;19:566-73. http://dx.doi.org/10.3201/eid1904.111799

25. Zhu P, van der Ende A, Falush D, Brieske N, Morelli G, Linz B, et al. Fit genotypes and escape variants of subgroup III Neisseria meningitidis during three pandemics of epidemic meningitis. Proc Natl Acad Sci U S A. 2001;98:5234-9. http://dx.doi.org/10.1073/ pnas.061386098

26. Taha MK, Giorgini D, Ducos-Galand M, Alonso JM. Continuing diversification of Neisseria meningitidis W135 as a primary cause of meningococcal disease after emergence of the serogroup in 2000. J Clin Microbiol. 2004;42:4158-63. http://dx.doi.org/10.1128/JCM.42.9.4158-4163.2004

27. Lamelas A, Harris SR, Röltgen K, Dangy JP, Hauser J, Kingsley RA, et al. Emergence of a new epidemic Neisseria meningitidis serogroup A clone in the African meningitis belt: high-resolution picture of genomic changes that mediate immune evasion. MBio. 2014;5:e1974-14. http://dx.doi.org/10.1128/ mBio.01974-14
28. Zhu B, Fan Y, Xu Z, Xu L, Du P, Gao Y, et al. Genetic diversity and clonal characteristics of ciprofloxacin-resistant meningococcal strains in China. J Med Microbiol. 2014;63:1411-8. http://dx.doi.org/10.1099/jmm.0.078600-0

29. Jolley KA, Wilson DJ, Kriz P, McVean G, Maiden MC. The influence of mutation, recombination, population history, and selection on patterns of genetic diversity in Neisseria meningitidis. Mol Biol Evol. 2005;22:562-9. http://dx.doi.org/10.1093/molbev/msi041

30. Didelot X, Maiden MC. Impact of recombination on bacterial evolution. Trends Microbiol. 2010;18:315-22. http://dx.doi.org/ 10.1016/j.tim.2010.04.002

31. Vos M, Didelot X. A comparison of homologous recombination rates in bacteria and archaea. ISME J. 2009;3:199-208. http://dx.doi.org/10.1038/ismej.2008.93

32. Budroni S, Siena E, Dunning Hotopp JC, Seib KL, Serruto D, Nofroni C, et al. Neisseria meningitidis is structured in clades associated with restriction modification systems that modulate homologous recombination. Proc Natl Acad Sci U S A. 2011;108:4494-9. http://dx.doi.org/10.1073/pnas.1019751108

33. Kong Y, Ma JH, Warren K, Tsang RS, Low DE, Jamieson FB, et al. Homologous recombination drives both sequence diversity and gene content variation in Neisseria meningitidis. Genome Biol Evol. 2013;5:1611-27. http://dx.doi.org/10.1093/gbe/evt116

34. He B, Jia Z, Zhou H, Wang Y, Jiang X, Ma H, et al. CC4821 serogroup $\mathrm{W}$ meningococcal disease in China. Int J Infect Dis. 2014;29:113-4. http://dx.doi.org/10.1016/j.ijid.2014.08.022

35. Lucidarme J, Lekshmi A, Parikh SR, Bray JE, Hill DM, Bratcher HB, et al. Frequent capsule switching in 'ultra-virulent' meningococci: are we ready for a serogroup B ST-11 complex outbreak? J Infect. 2017;75:95-103. http://dx.doi.org/10.1016/j.jinf.2017.05.015

36. Mustapha MM, Marsh JW, Krauland MG, Fernandez JO, de Lemos AP, Dunning Hotopp JC, et al. Genomic investigation reveals highly conserved, mosaic, recombination events associated with capsular switching among invasive Neisseria meningitidis serogroup W sequence type (ST)-11 strains. Genome Biol Evol. 2016;8:2065-75. http://dx.doi.org/10.1093/gbe/evw122

37. Chiou CS, Liao JC, Liao TL, Li CC, Chou CY, Chang HL, et al. Molecular epidemiology and emergence of worldwide epidemic clones of Neisseria meningitidis in Taiwan. BMC Infect Dis. 2006;6:25. http://dx.doi.org/10.1186/1471-2334-6-25

38. Nunes AM, Ribeiro GS, Ferreira IE, Moura AR, Felzemburgh RD, de Lemos AP, et al. Meningococcal carriage among adolescents after mass meningococcal c conjugate vaccination campaigns in Salvador, Brazil. PLoS One. 2016;11:e0166475. http://dx.doi.org/ 10.1371/journal.pone. 0166475

39. Moura ARSS, Kretz CB, Ferreira IE, Nunes AMPB, de Moraes JC, Reis MG, et al. Molecular characterization of Neisseria meningitidis isolates recovered from 11-19-year-old meningococcal carriers in Salvador, Brazil. PLoS One. 2017;12:e185038. http://dx.doi.org/10.1371/journal.pone.0185038

40. Kawasaki Y, Matsubara K, Takahashi H, Morita M, Ohnishi M, Hori $\mathrm{M}$, et al. Invasive meningococcal disease due to ciprofloxacinresistant Neisseria meningitidis sequence type 4821: the first case in Japan. J Infect Chemother. 2017 Dec 7:S1341-321X(17)30274-X [Epub ahead of print].

Address for correspondence: Min Chen, Shanghai Municipal Center for Disease Control and Prevention, 1380 W ZhongShan Rd, Shanghai, China; email: chenmin@scdc.sh.cn; Yohei Doi, Division of Infectious Diseases, University of Pittsburgh School of Medicine, S829 Scaife Hall, 3550 Terrace St, Pittsburgh, PA 15261, USA; email: yod4@pitt.edu; Minggui Wang, Institute of Antibiotics, Huashan Hospital, Fudan University, 12 M Wulumuqi Rd, Shanghai 200040, China; email: mgwang@fudan.edu.cn 